\title{
The elastic displacement and stress in a material to promote reflection and transmission of an incoming wave
}

\author{
Christine Marie Kennefick
}

Received: 7 January 2008/Accepted: 26 June 2008/Published online: 20 July 2008

(C) Springer Science+Business Media, B.V. 2008

\begin{abstract}
The conditions on elastic displacement and stress in a material that will promote reflection and transmission of an incoming wave are calculated. It is found, for example, that to optimize reflection and transmission, scalar potentials of the displacement in the wave and in the material will be related to rotations in planes perpendicular and parallel to the direction of propagation to the wave. When a pulse is constructed and its path analyzed through short distances, it is shown that abrupt transitions in tension and compression in a material will maximize reflection of the pulse. When strain energy is minimized where reflection and refraction are to occur, differences in tension and compression become prominent again. Finally, an approximate volume of material is calculated for an electron to harness the restoring forces in a material to balance the energy lost in inelastic scattering.
\end{abstract}

Keywords Elastic stress - Pulse ·

Transmission $\cdot$ Reflection $\cdot$ Strain energy

\section{Introduction}

The importance of controlling wave and crack propagation by designing the texture and geometry

C. M. Kennefick ( $\square)$

Department of Physics, Howard University, 2355 Sixth Street NW, Washington, DC 20059, USA

e-mail: ckennefick@howard.edu of the medium containing the wave is evident from both very recent findings and those in the past. It is the purpose of this paper to show relations between the displacement of an elastic stress wave and the displacement induced by a microstructural pattern that would enhance reflection or transmission of a wave.

Other recent studies for wave propagation and crack advance have utilized displacement, wave equations and finite elements. The propagation of waves in microstretch elastic solids, for example (Tumar and Khurana 2008), has been investigated by using vectors representing displacement and microrotation and by expressing these vectors as a sum of scalar and vector potentials. On a larger scale, with a packed array of circular rings, wave equations have been developed from a force balance for displacement and for rotation of a cross section (Shim et al. 2008). Fracture along grain boundaries, with a criterion involving an average normal tensile stress on a grain, has been numerically modeled using a Voronoi cell hybrid finite element method (Gastaldi et al. 2008).

The reflection and refraction of a pulse treated in this paper also complements previous studies that have analyzed the transmission of waves at bimaterial interfaces (Singh and Kavaturu 1997; Chattopadhyay 2004) and at interfaces composed of a frictional contact between two materials (Wang et al. 2004; Miller and Tran 1979, 1981; Cominou et al. 1979). Evidence for geometric arrangements affecting the speed, reflection and refraction of a pulse have been 
brought out in studies involving periodic and nonperiodic arrangements of particles embedded in a material (Geng et al. 2003; Kinra et al. 1998, 1999; Krasil'nikov 1999) and in a study involving oscillation of the speed of a crack tip (Blumenfeld 1998).

If particles and grains have elongated shapes, they can undergo a slight rotation within a material under an applied load. Such a rotation can give rise to elastic stresses in the matrix material that alternate spatially between tension and compression (Muskhelishvili 1953; Kennefick 2005, 2008). One purpose of this paper is to find a mapping of such alternating stresses so that a pulse is optimally reflected or transmitted in the material.

It is proposed that under another incoming tensile or compressive force, such as that provided by a mechanical or electrical pulse, the atoms in the material matrix will in turn create a temporary restoring force as they move toward their equilibrium bond positions. These temporary restoring forces would provide a positive or negative acceleration to the incoming pulse itself. Moreover, the change in speed of the pulse, particularly that occurring at an abrupt change from tension to compression in the atomic lattice, is expected to give rise to reflection and refraction of the pulse.

This paper also constructs a pulse that could emanate near the tip of crack under an applied load. The portions of a pulse that could refract and reflect as the pulse encounters a transition from tension to compression in the material are also derived. Finally, the approximate length scale in a material microstructure to have an electron wave packet reflect and transmit through a material by a similar mechanism is also calculated.

\section{The disturbance as a wave}

2.1 The propagation equation and the form of its solution

The incoming wave disturbance is assumed to cause a displacement $\vec{s}$ in the material. The displacement $\vec{s}$ is general, and can be as small as the displacement of electrons around atoms in a material, or can be a larger displacement involving many grains. At the same time, the incoming wave becomes subjected to the force $\vec{F}_{m}$ provided by the material microstructure.
The force $\vec{F}_{m}$ can arise, for example, from stress distributions from the elastic rotation of grains.

The notation of the operators used in Eq. 1 below and in other equations in this paper is the following. $\hat{i}$ is a unit vector in the $x$ direction, $\hat{j}$ is a unit vector in the $y$ direction and $\hat{k}$ is a unit vector in the $z$ direction. $\nabla$ is the operator $\hat{i} \frac{\partial}{\partial x}+\hat{j} \frac{\partial}{\partial y}+\hat{k} \frac{\partial}{\partial z}$, which can operate from the left upon the vector that follows it, or from the right on the vector that precedes it. On the left hand side of Eq. 1, the symbol - represents the scalar product between the two vectors just to the left and right of the symbol. The symbol $\times$ denotes a vector product. Finally, the two dots above the last term of Eq. 1 represent a second derivative with respect to time.

The propagation equation governing $\vec{s}$ for the incoming wave and the force $\vec{F}_{m}$ in the material is given by Nadeau (1964).

$a b c[(\lambda+2 \mu) \nabla \nabla \cdot \vec{s}-\mu \nabla \times(\nabla \times \vec{s})]+\vec{F}_{m}=m \ddot{\vec{s}}$

In the Eq. 1 above, $\lambda$ is an elastic constant equal to $v \mathrm{E} /[(1+v)(1-2 v)]$, where $v$ is Poisson's ratio and $\mathrm{E}$ is the Young's modulus for the material containing the wave. $\mu$ is the shear modulus of the material and $\mathrm{m}$ is a small amount of mass being displaced by the incoming wave.

The term abc at the front of the first term represents a small volume element containing the mass $\mathrm{m}$. The volume is written as a product of the dimensions $\mathrm{a}, \mathrm{b}$ and $\mathrm{c}$ of a rectangular shaped volume to provide some generality to the volume. Equation 1 is actually the sum of three independent equations representing forces in three directions along the orthogonal axes used. In each direction, the small volume, as well as the mass $m$ contained in it, can be different from the volume for displacement in another direction. Furthermore, $\vec{F}_{m}$ is the sum of forces in each direction, where each of these forces arise from stresses acting upon different planes. Later in this study, the small area in one of these planes is denoted by $\mathrm{ab}$, where the dimensions $\mathrm{a}$ and $\mathrm{b}$ can pertain to the area in that plane. The dimensions $a$ and $b$ can differ in another plane, as long as the area for the stress and the volume in Eq. 1 is kept small. To retain this flexibility and generality in Eq. 1, the volume is written as a product of the dimensions $\mathrm{a}, \mathrm{b}$ and $\mathrm{c}$, rather than having it be a volume of fixed dimensions in each direction. 
It is also recognized that when the element of volume is small enough, the Young's modulus E will vary in different crystallographic directions. These crystallographic directions themselves might not coincide with the coordinate directions used in Eq. 1, but a component of $\mathrm{E}$ in various crystallographic directions could contribute to each coordinate direction. When working at a small length scale in a single direction, therefore, these different values of $\mathrm{E}$ can be taken into account. To keep the notation simple in Eq. 1 and in the equations that follow, however, $\mathrm{E}$ and $\mu$ are written as a single symbol.

For the propagating wave, the displacement $\vec{s}$ can be written as the sum of a scalar potential $\psi$ and a vector potential $\vec{A}$ (Nadeau 1964). Thus $\vec{s}$ may be written as

$\vec{s}=\nabla \psi+(\nabla \times \vec{A})$.

In Eq. 2, $\psi$ can be written as the sum of three separate functions in the $\mathrm{x}, \mathrm{y}$ and $\mathrm{z}$ directions that are dependent upon time $t$ through a function $\mathrm{B}(\mathrm{t})$ :

$\Psi=[\mathrm{f}(\mathrm{x})+\mathrm{g}(\mathrm{y})+\mathrm{h}(\mathrm{z})] \mathrm{B}(\mathrm{t})$.

Similarly, the vector $\vec{A}$ in Eq. 2 can be written as the sum of three vectors in the $\mathrm{x}, \mathrm{y}$ and $\mathrm{z}$ directions with a time dependence given through the function $\mathrm{B}(\mathrm{t})$ :

$\vec{A}=[F \hat{i}+G \hat{j}+H \hat{k}] B(t)$.

Each function F, G and $\mathrm{H}$ in Eq. 4 is a function of the coordinates $x, y$ and $z: F=F(x, y, z), G=G(x, y, z)$ and $\mathrm{H}=\mathrm{H}(\mathrm{x}, \mathrm{y}, \mathrm{z})$. For simplicity in the analysis that follows, it will be assumed that the time dependence of the scalar and vector potentials of the incoming wave are the same, although this need not be the case.

The force $\vec{F}_{m}$ in the material that can interact with the wave is derived from the elastic stress tensor $\mathbf{T}_{\mathrm{m}}$, which in turn is related to the elastic strain tensor $\mathbf{S}_{\mathrm{m}}$ and to the elastic displacement $\vec{s}_{m}$. The displacement $\vec{s}_{m}$ in the material can also be written as a sum of a scalar potential and a vector potential:

$\vec{s}_{m}=\nabla \psi_{m}+\left(\nabla \times \vec{A}_{m}\right)$.

Similar to the function $\psi$ for the wave, the function $\psi_{\mathrm{m}}$ is a scalar function that is a sum of three functions $\mathrm{u}$, $\mathrm{v}$ and $\mathrm{w}$ that are each functions of $\mathrm{x}, \mathrm{y}$ and $\mathrm{z}$ respectively:

$\psi_{m}=[u(x)+v(y)+w(z)] D(t)$.

It is emphasized here that $\mathrm{u}, \mathrm{v}$ and $\mathrm{w}$ are a part of $\psi_{\mathrm{m}}$ and are not individually the total displacements in the $\mathrm{x}, \mathrm{y}$ and $\mathrm{z}$ directions. The function $\mathrm{D}(\mathrm{t})$ represents the dependence of $\psi_{\mathrm{m}}$ on time t.

Similar to $\vec{A}$, the vector potential of the wave disturbance, $\vec{A}_{m}$ is the sum of three vectors in the $\mathrm{x}, \mathrm{y}$ and $\mathrm{z}$ directions:

$\vec{A}_{m}=[U \hat{i}+V \hat{j}+W \hat{k}] D(t)$.

In Eq. 7 above, $\mathrm{U}, \mathrm{V}$ and $\mathrm{W}$ are all functions of $\mathrm{x}, \mathrm{y}$ and $\mathrm{z}$ : $\mathrm{U}$ is $\mathrm{U}(\mathrm{x}, \mathrm{y}, \mathrm{z}), \mathrm{V}$ is $\mathrm{V}(\mathrm{x}, \mathrm{y}, \mathrm{z})$, and $\mathrm{W}$ is $\mathrm{W}(\mathrm{x}, \mathrm{y}, \mathrm{z})$.

For example, $\mathrm{U}, \mathrm{V}$ and $\mathrm{W}$ can be functions of the distances in the $\mathrm{x}, \mathrm{y}$ and $\mathrm{z}$ directions from an apex of a three dimensional crystalline grain. If the coordinates of the apex are $\left(\mathrm{x}_{0}, \mathrm{y}_{0}, \mathrm{z}_{0}\right)$, and $\mathrm{U}_{1}, \mathrm{U}_{2}, \mathrm{U}_{3}, \mathrm{~V}_{1}$, $\mathrm{V}_{2}, \mathrm{~V}_{3}, \mathrm{~W}_{1}, \mathrm{~W}_{2}$, and $\mathrm{W}_{3}$ are numerical coefficients, $\mathrm{U}, \mathrm{V}$ and $\mathrm{W}$ can be written as (Kennefick 2008).

$$
\begin{aligned}
& U=\frac{U_{1} x_{0}^{2}}{x_{0}+\left|x-x_{0}\right|}+\frac{U_{2} y_{0}^{2}}{y_{0}+\left|y-y_{0}\right|}+\frac{U_{3} z_{0}^{2}}{z_{0}+\left|z-z_{0}\right|} \\
& V=\frac{V_{1} x_{0}^{2}}{x_{0}+\left|x-x_{0}\right|}+\frac{V_{2} y_{0}^{2}}{y_{0}+\left|y-y_{0}\right|}+\frac{V_{3} z_{0}^{2}}{z_{0}+\left|z-z_{0}\right|} \\
& W=\frac{W_{1} x_{0}^{2}}{x_{0}+\left|x-x_{0}\right|}+\frac{W_{2} y_{0}^{2}}{y_{0}+\left|y-y_{0}\right|}+\frac{W_{3} z_{0}^{2}}{z_{0}+\left|z-z_{0}\right|}
\end{aligned}
$$

The force $\vec{F}_{m}$ can be found from the stress tensor $\mathbf{T}_{\mathrm{m}}$, the vector $\vec{N}$ normal to a plane of material that interfaces with the incoming wave, and an area over which the stress acts. If this area is modeled as a rectangle of sides of length a and $b$, then the area over which the material stress acts has magnitude $a b$ and

$\vec{F}_{m}=\left(\vec{N} \cdot T_{m}\right) a b$.

The vector $\vec{N}$ has direction cosines with the $\mathrm{x}, \mathrm{y}$ and $\mathrm{z}$ axes of $\cos \delta, \cos \phi$, and $\cos \varphi$ and can be written

$\vec{N}=\hat{i} \cos \delta+\hat{j} \cos \varphi+\hat{k} \cos \phi$.

The stress tensor $\mathbf{T}_{\mathrm{m}}$ is related to the strain tensor $\mathbf{S}_{\mathrm{m}}$ by the relation (Nadeau 1964).

$\mathbf{T}_{m}=\mu \mathbf{S}_{m}+\lambda\left|\mathbf{S}_{m}\right| \mathbf{I}$

where $\mathbf{I}$ is an identity matrix, with the property that $\vec{V} \cdot \mathbf{I}=\mathbf{I} \cdot \vec{V}=\vec{V}$ for any vector $\vec{V}$. The stress tensor $\mathbf{S}_{\mathrm{m}}$ in Eq. 13 is related to the displacement $\vec{s}_{m}$ in the material by

$\mathbf{S}_{m}=\frac{\nabla \overrightarrow{s_{m}}+\overrightarrow{s_{m}} \nabla}{2}$. 
In Eq. 13, the quantity $\left|S_{m}\right|$ is the trace of the tensor $\mathbf{S}_{\mathrm{m}}$, which can be calculated as the square of the quantity $\nabla \cdot \vec{s}_{m}$ or as S:I. Here the symbol : denotes a double scalar product. For the operator :, the upper symbol represents the scalar product between the second vector of the first tensor and the first vector of the second tensor. The lower symbol denotes the scalar product between the first vector of the first tensor and the second vector of the second tensor.

Combining Eqs. 5, 6, 7, 11, 13 and 14 gives

$$
\begin{aligned}
\vec{F}_{m}= & a b \vec{N} \cdot\left\{\mu \left[\nabla \nabla \psi+\frac{\nabla\left(\nabla \times \vec{A}_{m}\right)}{2}\right.\right. \\
& \left.\left.+\frac{\left.\left(\nabla \times \vec{A}_{m}\right) \nabla\right]}{2}\right]+\lambda \nabla \cdot \nabla\left(\psi_{m} \mathbf{I}\right)\right\} .
\end{aligned}
$$

Combining Eqs. 1, 2 and 15 gives for the propagation equation

$$
\begin{aligned}
& m \frac{\partial^{2}}{\partial t^{2}}[\nabla \psi+(\nabla \times \vec{A})] \\
& =a b c\{(\lambda+2 \mu) \nabla \nabla \cdot[\nabla \psi+(\nabla \times \vec{A})] \\
& \quad-\mu \nabla \times \nabla \times[\nabla \psi+(\nabla \times \vec{A})]\} \\
& +a b \vec{N} \cdot\left\{\mu \left[\nabla \nabla \psi_{m}+\frac{\nabla\left(\nabla \times \vec{A}_{m}\right)}{2}\right.\right. \\
& \left.\left.+\frac{\left.\left(\nabla \times \vec{A}_{m}\right) \nabla\right]}{2}\right]+\lambda \nabla \cdot \nabla\left(\psi_{m} 1\right)\right\} .
\end{aligned}
$$

It will be assumed that an incoming wave will have a particular form and that $\psi$ and $\vec{A}$ are fixed and known. Hence the solution to Eq. 16 and to related conditions for reflection and transmission consists of finding the forms of $\psi_{\mathrm{m}}$ and $\vec{A}_{m}$ and the relation of these functions to $\psi$ and $\vec{A}$.

\subsection{Equations of interaction}

Equation 16 can be reduced further by simplifying the operator $\nabla \times \nabla \times$ to $(\nabla \nabla \cdot)-(\nabla \cdot \nabla)$, and recognizing that the operator $\nabla \times \nabla$ on an argument yields zero. Then one can combine all the terms for the incoming wave on one side of the equation and all the terms for the material on the other. The result of these two processes gives

$$
\begin{aligned}
m & \frac{\partial^{2}}{\partial t^{2}}[\nabla \psi+(\nabla \times \vec{A})] \\
& -a b c\{(\lambda+2 \mu) \nabla \nabla \cdot \psi+\mu \nabla \cdot \nabla \nabla \psi \\
& +\mu \nabla \times(\nabla \cdot \nabla \vec{A})\} \\
= & a b \vec{N} \cdot\left\{\mu \nabla \nabla \psi_{m}+\lambda \nabla \cdot \nabla\left(\psi_{m} 1\right)\right. \\
& \left.+\frac{\nabla\left(\nabla \times \vec{A}_{m}\right)}{2}+\frac{\left(\nabla \times \vec{A}_{m}\right) \nabla}{2}\right\} .
\end{aligned}
$$

Combining Eqs. 3, 4, 6, 7, 12 and 17 and equating the $\hat{i}, \hat{j}$ and $\hat{k}$ components on each side of the resulting equation separately yields three equations. Each of these three equations in turn can be separated into terms involving $\partial / \partial x, \partial / \partial y$ and $\partial / \partial z$. When the sum of the terms involving each partial derivative $\partial / \partial x$, $\partial / \partial y$ and $\partial / \partial z$ are set to zero, three more equations are obtained that must hold true for the propagation equation 17. An example of three of these equations for propagation in the $\hat{i}$ direction is given below.

$$
\begin{aligned}
& m B^{\prime \prime}(t) f+a b c B(t)\left[(\lambda+\mu)\left(f_{x x}+g_{y y}+h_{z z}\right)\right. \\
& \left.\quad+\mu\left(f_{x x}+f_{y y}+f_{z z}\right)\right] \\
& =-a b D(t)\left[(\lambda+\mu) u_{x} \cos \delta+\mu\left(u_{y} \cos \phi+u_{z} \cos \phi\right)\right. \\
& \quad-\frac{\mu a b D(t)}{2}\left[2\left(W_{y}-V_{z}\right) \cos \delta+\left(U_{z}-W_{x}\right) \cos \phi\right. \\
& \left.\quad+\left(V_{x}-U_{y}\right) \cos \varphi\right], \\
& m B^{\prime \prime}(t) H+a b c \mu B(t)\left(H_{x x}+H_{y y}+H_{z z}\right) \\
& \quad=-\lambda a b D(t) v_{y} \cos \delta-\frac{\mu a b D(t)}{2}\left(W_{y}-V_{z}\right) \cos \phi,
\end{aligned}
$$

$$
\begin{aligned}
& m B^{\prime \prime}(t) G+a b c \mu B(t)\left(G_{x x}+G_{y y}+G_{z z}\right) \\
& \quad=\lambda a b D(t) w_{z} \cos \delta+\frac{\mu a b D(t)}{2}\left(W_{y}-V_{z}\right) \cos \phi .
\end{aligned}
$$

In Eqs. 18-20 and those that follow, the symbol $B^{\prime \prime}$ denotes the second derivative of $B$ with respect to time. A single prime symbol following a function denotes a first derivative. Subscripts involving $\mathrm{x}$, $\mathrm{y}$ or 
$\mathrm{z}$ are partial derivatives with respect to $\mathrm{x}, \mathrm{y}$ or $\mathrm{z}$. A single subscript is a first partial derivative, whereas a double subscript is a second partial derivative with respect to that variable.

The last three terms in Eq. 18 show that the displacement functions of the incoming wave will interact with rotation within the atomic planes of the material. The last terms in Eqs. 19 and 20 show out of plane rotations in which the direction of the rotation itself is in a plane perpendicular to the $\hat{i}$ direction of propagation. In this study, "in-plane" and "out-ofplane" refer to actual planes of atoms having the normal vector $\vec{N}$.

\subsection{Reflection}

One condition for maximizing the reflection of the incoming wave could be to maximize the acceleration of the wave in the opposite direction. Suppose $\vec{p}$ is a vector representing the direction of propagation of the wave. If the angles $\alpha, \beta$ and $\gamma$ are the angles this propagation vector make with the $\mathrm{x}, \mathrm{y}$ and $\mathrm{z}$ axes, then

$\vec{p}=\hat{i} \cos \alpha+\hat{j} \cos \beta+\hat{k} \cos \gamma$.

Further, let the vector $\vec{L} / \mathrm{m}$ represent an acceleration of the wave as it traverses through the material. Then from Eqs. 1 and $16 \vec{L}$ can be written as

$$
\begin{aligned}
\vec{L}= & -(\lambda+\mu) a b c \nabla \nabla \cdot \nabla \psi-\mu a b c \nabla \cdot \nabla \nabla \psi-\mu a b c \nabla \\
& \times(\nabla \cdot \nabla \vec{A})-\mu a b \vec{N} \cdot \nabla \nabla \psi_{m}-\lambda a b \vec{N} \cdot\left(\nabla \cdot \nabla \psi_{m}\right) \\
& \times 1-\frac{\mu a b \vec{N}}{2} \cdot\left[\nabla\left(\nabla \times \vec{A}_{m}\right)+\left(\nabla \times \vec{A}_{m}\right) \nabla\right] .
\end{aligned}
$$

For reflection, if this acceleration per unit mass is to be maximized in the direction of $-\vec{p}$, then one can write as one necessary condition that

$\nabla \vec{L} \cdot(-\vec{p})=0$.

Equations 3, 4, 6 and 7 were combined with Eqs. 22 and 23 to give relations between the components of displacement of the incoming wave and the components of the displacement in the material. With this combination of equations, Eq. 23 can be solved by collecting all terms in the $\mathrm{x}, \mathrm{y}$ and $\mathrm{z}$ directions separately. Each total function in the $\hat{i}, \hat{j}$ and $\hat{k}$ directions can then be set into three functions, each being the argument of the operator $\partial^{2} / \partial x^{2}, \partial^{2} / \partial x \partial y$ or $\partial^{2} / \partial x \partial z$. Setting these last three functions to zero in each of three directions will give nine equations. A sample of three of these equations derived from terms originally collected in the $\hat{i}$ direction is given below.

$$
\begin{aligned}
a b c B(t)\left[(\lambda+\mu)\left(f_{x x}+g_{y y}+h_{z z}\right)\right. \\
\left.\quad+\mu\left(f_{x x}+f_{y y}+f_{z z}\right)\right] \cos \alpha+\mu a b c B(t) \frac{\partial}{\partial x}\left[\left(H_{y}-G_{z}\right)\right. \\
\left.\quad \times \cos \alpha+\left(F_{z}-H_{x}\right) \cos \beta+\left(G_{x}-F_{y}\right) \cos \gamma\right] \\
=-\mu a b D(t)\left[u_{x}(2 \cos \delta \cos \alpha+\cos \phi \cos \beta\right. \\
\left.\quad+\cos \varphi \cos \gamma)+v_{y} \cos \delta \cos \beta+w_{z} \cos \delta \cos \gamma\right] \\
-\mu a b D(t)\left[\left(W_{y}-V_{z}\right) \cos \delta+\frac{\left(U_{z}-W_{x}\right)}{2} \cos \phi\right. \\
\left.+\frac{\left(V_{x}-U_{y}\right)}{2} \cos \varphi\right] \cos \alpha-\mu a b D(t)\left[\frac{\left(U_{z}-W_{x}\right)}{2}\right. \\
\left.\quad \times \cos \delta \cos \beta+\frac{\left(V_{x}-U_{y}\right)}{2} \cos \delta \cos \gamma\right]
\end{aligned}
$$

$a b c B(t)\left[(\lambda+\mu)\left(f_{x x}+g_{y y}+h_{z z}\right)\right.$

$\left.+\mu\left(g_{x x}+g_{y y}+g_{z z}\right)\right] \cos \beta$

$+\mu a b c B(t) \frac{\partial}{\partial y}\left[\left(H_{y}-G_{z}\right) \cos \alpha\right.$

$\left.+\left(F_{z}-H_{x}\right) \cos \beta+\left(G_{x}-F_{y}\right) \cos \gamma\right]$

$=-\mu a b D(t)\left[v_{y}(2 \cos \phi \cos \beta+\cos \delta \cos \alpha\right.$

$\left.+\cos \varphi \cos \gamma)+u_{x} \cos \phi \cos \alpha+w_{z} \cos \phi \cos \gamma\right]$

$-\mu a b D(t)\left[\left(U_{z}-W_{x}\right) \cos \phi+\frac{\left(V_{x}-U_{y}\right)}{2} \cos \varphi\right.$

$\left.+\frac{\left(W_{y}-V_{z}\right)}{2} \cos \delta\right] \cos \beta-\mu a b D(t)\left[\frac{\left(W_{y}-V_{z}\right)}{2}\right.$

$\left.\times \cos \phi \cos \alpha+\frac{\left(V_{x}-U_{y}\right)}{2} \cos \phi \cos \gamma\right]$

$\operatorname{abcB}(t)\left[(\lambda+\mu)\left(f_{x x}+g_{y y}+h_{z z}\right)\right.$

$\left.+\mu\left(h_{x x}+h_{y y}+h_{z z}\right)\right] \cos \gamma$

$+\mu a b c B(t) \frac{\partial}{\partial z}\left[\left(H_{y}-G_{z}\right) \cos \alpha\right.$

$\left.+\left(F_{z}-H_{x}\right) \cos \beta+\left(G_{x}-F_{y}\right) \cos \gamma\right]$

$=-\mu a b D(t)\left[w_{z}(2 \cos \varphi \cos \gamma+\cos \delta \cos \alpha\right.$

$\left.+\cos \phi \cos \beta)+u_{z} \cos \varphi \cos \alpha+v_{y} \cos \varphi \cos \beta\right]$

$-\mu a b D(t)\left[\left(V_{x}-U_{y}\right) \cos \varphi+\frac{\left(W_{y}-V_{z}\right)}{2} \cos \delta\right.$ 


$$
\begin{aligned}
& \left.+\frac{\left(U_{z}-W_{x}\right)}{2} \cos \phi\right] \cos \gamma \\
& -\mu a b D(t)\left[\frac{\left(W_{y}-V_{z}\right)}{2} \cos \varphi \cos \alpha\right. \\
& \left.+\frac{\left(U_{z}-W_{x}\right)}{2} \cos \varphi \cos \beta\right]
\end{aligned}
$$

What is particularly new in Eqs. 24 to 26 that is not shown in Eqs. 20 to 22 for general propagation is that for reflection, the spatial variation of the rotations produced by the incoming wave become related to rotations in the same plane of the material. The rotations this same plane can be either in plane or out of plane of the material lattice.

\subsection{Transmission}

There are two conditions that might hold for optimum transmission of a wave, particularly a conduction wave, through a material. One is that the force in the material superimposes with the force of the wave to produce a strain energy of zero in the very region occupied by the wave. The total strain energy per unit volume $\mathrm{u}_{\text {total }}$ can be written as (Nadeau 1964)

$u_{\text {total }}=\mu \mathbf{S}_{\text {total }}: \mathbf{S}_{\text {total }}+\frac{1}{2} \lambda\left|\mathbf{S}_{\text {total }}\right|^{2}$.

In Eq. 27 above, $\mathbf{T}_{\text {total }}$ is $\mathbf{T}+\mathbf{T}_{\mathrm{m}}$ and $\mathbf{S}_{\text {total }}$ is $\mathbf{S}+\mathbf{S}_{\mathrm{m}}$. The strain tensor $\mathbf{S}_{\text {total }}$ can be derived from a total displacement $\vec{s}_{\text {total }}$ with the same Eq. 14, except $\vec{s}_{\text {total }}$ replaces $\vec{s}_{m}$. Here

$\vec{s}_{\text {total }}=\nabla\left(\psi+\psi_{m}\right)+\nabla \times\left(\vec{A}+\vec{A}_{m}\right)$.

Another possibility for optimum transmission of the wave is that the total strain energy per unit volume decreases as steeply as possible for each increment of advance of the wave in the direction of the propagation vector $\vec{p}$. The variation of the total strain energy in the direction of $\vec{p}$ would then be maximized. Then one necessary condition is that there be in inflection point in the variation of the total energy density in the direction of $\vec{p}$ :

$\nabla\left[\left(\nabla u_{\text {total }} \cdot \vec{p}\right)\right] \cdot \vec{p}=0$.

Equations 2 through 7 and 28 were combined separately with Eqs. 27 and 29. The resulting equations are too long to be reproduced here, but to satisfy either of the Eqs. 27 or 29, a sample of three relations that could be set to zero are shown below.

$$
\begin{aligned}
& \frac{\partial}{\partial x}\left[u_{x}+\left(W_{y}-V_{z}\right)\right]=0 \\
& {\left[f_{x x} B(t)+u_{x x} D(t)\right]^{2}=0} \\
& \left\{\frac{\partial}{\partial z}\left[B(t)\left(G_{x}-F_{y}\right)+D(t)\left(V_{x}-U_{y}\right)\right]\right\}^{2}=0
\end{aligned}
$$

Equation 30 suggests that for the stresses inside the material, the slope of one scalar potential in a particular direction must be equal to the negative of the rotation in a plane perpendicular to that particular direction. Equations 31 and 32 indicate that both the scalar potentials and the rotational modes of the material displacements must be 180 degrees out of phase with those of the incoming wave. Alternatively, the function in the interior of Eq. 32 can be written as $\left[B(t) G_{x}-D(t) U_{y}\right]+\left[D(t) V_{x}-B(t) F_{y}\right]$. This last expression implies that a specific coupling could exist between rotational displacement in the wave and that in the material to promote a strain energy of zero.

\section{The incoming wave as a pulse}

\subsection{Construction of the pulse}

In this section, the direction of propagation of the pulse will be in the $x$ direction, where $x, y, z$ is an orthogonal coordinate system local to and traveling with the stress pulse. The function $f(x)$ in this section will represent the strength of the stress in the pulse and will be in the y direction.

For the model of the pulse as a simple block function, $f(x)$ is a concentrated stress $\sigma_{c}$ along the length from $x$ equal to zero to $x$ equal to $L$, as shown in Fig. 1 .

The pulse itself can be constructed as the Fourier integral

$f(x)=\frac{2 \sigma_{c}}{\pi} \int_{0}^{\infty} \frac{\sin k L}{k} \cos k(x-v t) d k$.

The pulse can also be modeled as a simple sine function, as shown in Fig. 2. The form of this pulse might be suitable for a longitudinal pulse in which the leading part is compressive and the trailing part is tensile. In this case, the length of the disturbance is $L$, with the maximum concentrated stress $\sigma_{c}$ occurring at $L / 4$ and $3 L / 4$. 


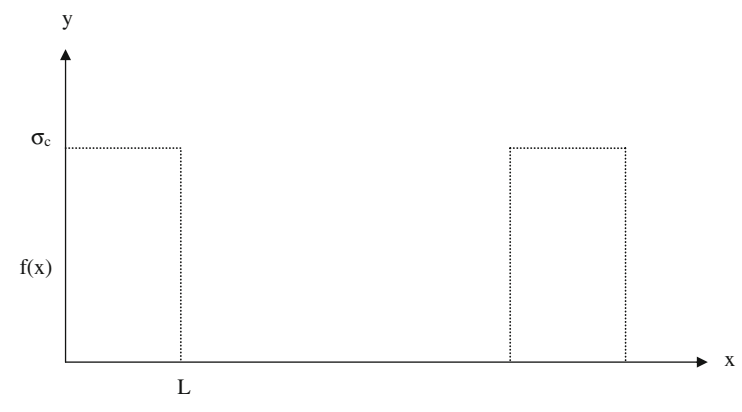

Fig. 1 The pulse as a block function traveling in the $x$ direction, with a maximum at a concentrated stress $\sigma_{\mathrm{c}}$

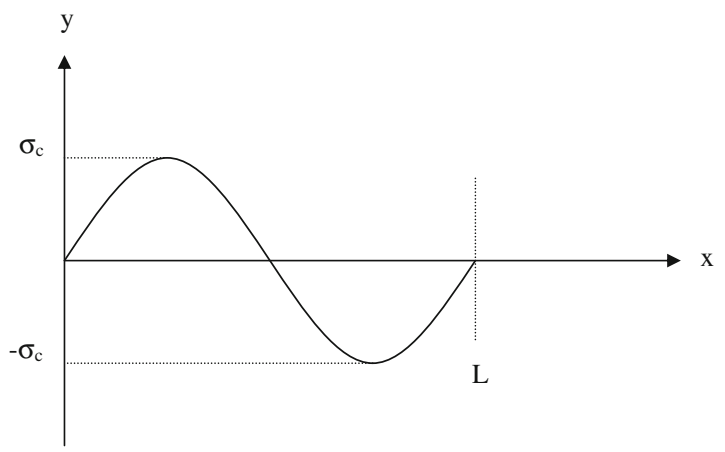

Fig. 2 The pulse as a sine function traveling in the $x$ direction. The leading maximum in the function is a compressive concentrated stress $\sigma_{\mathrm{c}}$. The traveling minimum in the pulse is a tensile concentrated stress $\sigma_{\mathrm{c}}$

Following the same procedures for constructing the previous Fourier integral for the block function for $\sigma_{c}$, the pulse in Fig. 2 can be expressed as

$f(x)=\int_{0}^{\infty} \frac{4 \sigma_{c} \sin k L \sin k(x-v t)}{4 \pi^{2}-L^{2} k^{2}} d k$.

\subsection{Optimum stress in the material for reflection} and refraction for short distances traveled by a pulse

In Eqs. 33 and 34, the term vt, the distance the pulse has traveled, is a function of time. It was desired to get the term $v t$ as a function of the local stress state $\sigma(x)$ in the material where the pulse is traveling. For the simple block function, this transformation was done by considering a very short distance near an interface between tension and compression in the material where the pulse would both reflect and refract due to an abrupt change in speed. For this short distance, the acceleration of the pulse was assumed constant as it traversed a short distance containing a constant restoring force in the material. Since the pulse accelerates under the restoring forces of the atoms in the material lattice, the term $v t$ can be replaced by a general distance variable $d$.

In the analysis in this section, $\sigma(x)$ is a stress state in the material matrix that can be tensile, compressive or shear. It can, for example, be the stress state from the rotation of material grains. The stress $\sigma(x)$ is a force per unit area, with $A$ denoting this unit area. The terms $\rho$ and $V$ are defined respectively as the mass per unit volume of the material and the unit volume of the material itself.

For some short length of time $t$, suppose the pulse can travel a short distance $d$ in the absence of stresses in the material. The pulse is under the influence of only the remote applied stress $\sigma_{\infty}$. The constant acceleration under this stress, denoted by the letter $a$, is then $\sigma_{\infty} A / \rho V$. Under this constant acceleration, the distance $d$ traveled is given by $a t^{2} /$ 2 , assuming that the change of speed sought is different from a constant speed in the expression vt, and that this initial relative speed is zero. From equating $\mathrm{d}$ with $a t^{2} / 2$, where the acceleration $\mathrm{a}$ is $\sigma_{\infty} A / \rho V$, the quantity $t^{2}$ can be obtained as $2 d \rho V /$ $\sigma_{\infty} A$.

By the preceding analysis, the acceleration $a_{m}$ of the pulse under the influence of the restoring forces of the atoms in the material during this same time interval $t$ is $\sigma(x) A / \rho V$, where $\sigma(x)$ is considered a constant for the short time $t$. The distance $d$, given by $a_{m} t^{2} / 2$, becomes $\sigma(x) A / \rho \mathrm{V}$ multiplied by $2 d \rho V / \sigma_{\infty} A$, which is $\sigma(x) d / \sigma_{\infty}$. The distance $d$ traveled in the same direction under the influence of material stresses is thus scaled by a ratio of the local stress in the material to the remote applied stress.

Suppose, for example, that one wants to maximize the reflection of an initial pulse so that a stress concentration does not build up in a material. With the $x, y, z$ coordinate axes kept local to the pulse with the direction of propagation in the $x$ direction, the initial pulse, a refracted pulse and a reflected pulse can all have the form of Eq. 33. The term $v t$ in this case has become the distance $\mathrm{d}$ just discussed. One would want to find the optimum pattern in $\sigma(x)$ to maximize this reflection. Substituting $\sigma(x) x / \sigma_{\infty}$ for $d$ 
in Eq. 33, which has become $v t$, taking the derivative of Eq. 33 with respect to $x$ and setting the result equal to zero would be a first step in maximizing the reflected pulse. A reflected pulse, in summary, would produce a maximum change in the presence of the pulse at positions $\mathrm{x}$ where the pulse is expected to be if it keeps moving forward.

The process just mentioned gives the following two possibilities for $\sigma(\mathrm{x})$ :

$\sigma(x)=\sigma_{\infty}\left(1-\frac{m \pi}{k x}\right) \quad \mathrm{m}$ an integer

and

$x \sigma^{\prime}(x)+\sigma(x)-\sigma_{\infty}=0$.

Equation 36 can be easily solved (Boyce and DiPrima 1977) to give the solution

$\sigma(x)=\sigma_{\infty}+\frac{C}{x} \quad \mathrm{x}>0$

for a constant $C$ and values of $x$ greater than zero in front of the pulse.

For reflection of the pulse to be maximized, both Eqs. 35 and 37 predict transitions, even abrupt transitions, between tensile and compressive stress states depending upon the values of the integer $m$ and the constant $C$.

\section{The pulse as a result of a large initial displacement}

\subsection{Introduction and notation}

In this section, an expression for a pulse is derived that is based upon a large initial displacement occurring in a material subjected to a disturbance. In the last two sections, in contrast, a constant concentrated stress $\sigma_{\mathrm{c}}$ essentially acted as a coefficient in front of the mathematical form of the pulse.

A large displacement, for example, could occur for those atoms near the tip of a crack, as shown schematically in Fig. 3. This displacement could possibly travel as an attenuating pulse of stress in the material, leaving behind a concentrated stress surrounding the tip of the crack. It is the purpose of this section to derive a full stress tensor for a pulse due to such a large initial displacement.

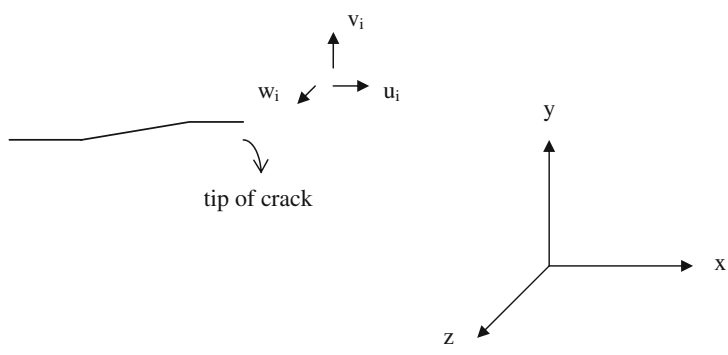

Fig. 3 A disturbance beginning at the point $\left(\mathrm{x}_{0}, \mathrm{y}_{0}, \mathrm{z}_{0}\right)$ causes an atom to displace by $\mathrm{u}_{\mathrm{i}}$ in the $x$ direction, by $\mathrm{v}_{\mathrm{i}}$ in the $\mathrm{y}$ direction and by $\mathrm{w}_{\mathrm{i}}$ in the $\mathrm{z}$ direction

Once the initial displacement occurs, it travels as a pulse in the $x, y$ and $z$ directions, as shown in Fig. 3. In each of the $x, y$ and $z$ directions, a component of the pulse will travel and will be modeled as a block or sine function, as calculated in Sect. 3. The maximum amplitude, however, will be based upon the displacements $u_{i}, v_{i}$ and $w_{i}$.

As the pulse travels into the material, it will be assumed that each of the initial displacements $u_{i}, v_{i}$ and $w_{i}$, along with its functional dependence upon $x$, $y$, and $z$, attenuates as $1 / x, 1 / y$ and $1 / z$. Such an attenuation might yield a radial distribution of stress around the end of a crack that is different from what has been previously modeled. The final form the stress components here, however, will show how the stress in the material from the $v t$ term in the pulse calculated in Sect. 3 could affect reflection and transmission of the pulse.

According to what has just been discussed in the preceding paragraphs, one form of the displacement vector $\vec{S}$ could be

$$
\begin{aligned}
\vec{S}= & \hat{i} \frac{u_{i} x_{0} y_{0} z_{0}}{x y z} P(x)+\hat{j} \frac{v_{i} x_{0} y_{0} z_{0}}{x y z} P(y) \\
& +\hat{k} \frac{w_{i} x_{0} y_{0} z_{0}}{x y z} P(z) .
\end{aligned}
$$

As discussed in Sect. 2.1 of this paper with Eq. 14, the strain tensor $\mathbf{S}$ can then be calculated from its definition of $(\nabla \vec{s}+\vec{s} \nabla) / 2$, and the stress tensor $\mathbf{T}$ can be found from the expression $2 \mu \mathbf{S}+\lambda|\mathbf{S}| \mathbf{I}|\mathbf{S}|$ in the analysis here is $S_{11}+S_{22}+S_{33}$, where $S_{11}, S_{22}$ and $S_{33}$ are the coefficients of the $\hat{i} \hat{i}, \hat{j} \hat{j}$ and $\hat{k} \hat{k}$ components respectively of the tensor $\mathbf{S}$. Finally, $\mathbf{I}$ is an identity tensor equal here to $\hat{i} \hat{i}+\hat{j} \hat{j}+\hat{k} \hat{k}$.

From the preceding discussion and Eq. 38, the stress tensor $\mathbf{T}$ can be written as 


$$
\begin{array}{r}
\hat{i i} \frac{x_{0} y_{0} z_{0}}{x y z}\left\{u_{i}(2 \mu+\lambda)\left[P^{\prime}(x)-(1 / x) P(x)\right]+\lambda\left[v_{i} P^{\prime}(y)+w_{i} P^{\prime}(z)-\left(v_{i} / y\right) P(y)-\left(w_{i} / z\right) P(z)\right]\right\} \\
+\hat{j} \hat{j} \frac{x_{0} y_{0} z_{0}}{x y z}\left\{v_{i}(2 \mu+\lambda)\left[P^{\prime}(y)-(1 / y) P(y)\right]+\lambda\left[u_{i} P^{\prime}(x)+w_{i} P^{\prime}(z)-\left(u_{i} / x\right) P(x)-\left(w_{i} / z\right) P(z)\right]\right\} \\
+\hat{k} \hat{k} \frac{x_{0} y_{0} z_{0}}{x y z}\left\{w_{i}(2 \mu+\lambda)\left[P^{\prime}(z)-(1 / z) P(z)\right]+\lambda\left[u_{i} P^{\prime}(x)+v_{i} P^{\prime}(y)-\left(u_{i} / x\right) P(x)-\left(v_{i} / y\right) P(y)\right]\right\} \\
-\mu \frac{x_{0} y_{0} z_{0}}{x y z}\left\{\begin{array}{r}
(\hat{i} \hat{j}+\hat{j} \hat{i})\left[\left(u_{i} / y\right) P(x)+\left(v_{i} / x\right) P(y)\right]+(\hat{i} \hat{k}+\hat{k} \hat{i})\left[\left(u_{i} / z\right) P(x)+\left(w_{i} / x\right) P(z)\right] \\
+(\hat{j} \hat{k}+\hat{k} \hat{j})\left[\left(v_{i} / z\right) P(y)+\left(w_{i} / y\right) P(z)\right]
\end{array}\right\}
\end{array}
$$

In Sect. 6, the Discussion section of this paper, it will be noted that the conditions on the terms $P^{\prime}(x)$, $P^{\prime}(y)$ and $P^{\prime}(z)$ in the equation above, in order to have the stresses not increase or oscillate without bound as $\mathrm{x}, \mathrm{y}$ and $\mathrm{z}$ become large, correlate with the conditions for reflection found in Sect. 3.2.

\section{Portions of the pulse that reflect and refract}

\subsection{Introduction and notation}

As a pulse changes speed due to the stresses and restoring forces in the material lattice, it is expected to reflect and refract. In this case, the reflection and refraction are not necessarily due to a change in material at an interface. The reflection and refraction are expected to occur at a transition between tension and compression in the matrix material.

Since the material here is being modeled as an interconnected network of atomic bonds, both the stress and displacement can be different across a row of atoms where the pulse reflects and refracts. Therefore, instead of a boundary condition that equates force or displacement across an interface, a condition that minimizes potential energy was used to find the fraction $p_{1}$ of the pulse that is reflected.

In this section, a part of the pulse traveling in the $x$ direction will be analyzed. The analysis will be similar for the parts of the initial pulse traveling in the $y$ and $z$ directions. It will be assumed that the incoming portions of the pulse involving the stress coefficients $\mathrm{T}_{11}, \mathrm{~T}_{12}$ and $\mathrm{T}_{13}$ of the tensor $\mathbf{T}$ in Eq. 39 will act independently. Here $T_{11}$ is the coefficient of the $\hat{i} \hat{i}$ term in Eq. 39, $\mathrm{T}_{12}$ the coefficient of the $\hat{i} \hat{j}$ term and $\mathrm{T}_{13}$ of the $\hat{i} \hat{k}$ term, with similar correspondences for the other coefficients. The numeral 1 denotes the $x$ direction, 2 denotes the $y$ direction and 3 the $z$ direction. The first subscript on the coefficient $\mathrm{T}$ denotes the direction of a vector normal to the plane upon which the force acts. The second subscript denotes the direction of the force itself. Hence $T_{11}$ will denote a longitudinal pulse and $\mathrm{T}_{12}$ and $\mathrm{T}_{13}$, which are the same as $T_{21}$ and $T_{31}$ in a symmetric strain tensor, will denote pulses of shear stress traveling in the same $x$ direction but in planes orthogonal to one another.

When the pulse reflects, the strain energy per unit volume to be minimized is given by $(1 / 2) \mathbf{T}: \mathbf{S}$ (Nadeau 1964). When working with a stress tensor $\mathbf{T}$, it is also useful to calculate strain energy from various stress components from the equation (1/ $2 E)\left[(1+v) \mathbf{T}: \mathbf{T}-v|\mathbf{T}|^{2}\right]$.

\subsection{Superposition and portion of the pulse that reflects}

For the part of the pulse containing the shear stress $\mathrm{T}_{12}$, for example, the portion that is reflected is $p_{1} \mathrm{~T}_{12}$. The portion of the pulse undergoing refraction is then $\left(1-p_{1}\right) \mathrm{T}_{12}$. It was also assumed that the portion of reflected stress $p_{1} \mathrm{~T}_{12}$ is superimposed with the stress already in the material in the region where the reflected pulse travels. Hence the $x, y, z$ coordinate system for $\mathrm{T}_{12}$ is local to the direction of travel of the pulse and is the same as the coordinate system for the 
stress $\mathrm{T}_{12}{ }^{\mathrm{ml}}$ in the material. For $\mathrm{T}_{12}{ }^{\mathrm{ml}}, \mathrm{m}$ is a label denoting a stress intrinsic to the material and 1 represents a region of reflection. Similar to the region of the reflected pulse, the stress in the region of material where the refracted portion travels was labeled $\mathrm{T}_{12} \mathrm{mr}$.

For this calculation, it was assumed that the incoming pulse is approximately the same magnitude as the stress in the regions where the pulse could get reflected or refracted. Situations in which the incoming pulse is much larger than or smaller than the stresses in the material are discussed in the next section.

From the definitions of $\mathrm{T}_{12}, \mathrm{p}_{1}, \mathrm{~T}_{12}{ }^{\mathrm{ml}}$ and $\mathrm{T}_{12}{ }^{\mathrm{mr}}$ given in this section and from the final equation for strain energy given in the last section, the strain energy per unit volume in the region of the reflected part of the pulse due to the superposition of the incoming stress pulse and the stress already in the material is $(1+v) /\left[E\left(\mathrm{p}_{1} \mathrm{~T}_{12}+\mathrm{T}_{12}{ }^{\mathrm{ml}}\right)^{2}\right]$. Similarly, the strain energy per unit volume in the region of material where the refracted portion travels is $(1+v) /\left\{E\left[\left(1-p_{1}\right) \mathrm{T}_{12}+\mathrm{T}_{12}{ }^{\mathrm{mr}}\right]^{2}\right\}$. The sum of the strain energy per unit volume in both the regions where reflection and refraction could occur was then labeled $\mathrm{U}$ and is given by

$U=\frac{(1+v)}{E}\left\{\frac{1}{\left(p_{l} T_{12}+T_{12}{ }^{m l}\right)^{2}}+\frac{1}{\left[\left(1-p_{l}\right) T_{12}+T_{12}{ }^{m r}\right]^{2}}\right\}$.

For a minimum in total strain energy per unit volume $U$, its derivative with respect to $p_{l}$ was taken. Setting this derivative equal to zero and solving for $p_{1}$ for a minimum in strain energy then gives

$p_{1}=\frac{T_{12}+T_{12}{ }^{m r}-T_{12}{ }^{m l}}{2 T_{12}}$.

A similar procedure involving the longitudinal pulse associated with $\mathrm{T}_{11}$ gives the same kind of expression for the portion of the pulse that is reflected. The result for the portion $p_{1}$ that is reflected is

$p_{1}=\frac{T_{11}+T_{11}{ }^{m r}-T_{11}{ }^{m l}}{2 T_{11}}$.

In Eqs. 41 and 42 above, it is evident that differences in positive and negative quantities in $\mathrm{T}^{\mathrm{mr}}$ and $\mathrm{T}^{\mathrm{ml}}$ will allow both the portions $p_{1}$ and $1-p_{1}$ to vary to their greatest extent.
5.3 Incoming stress pulse much smaller or larger than the stresses in the material

If the incoming stress pulse is much smaller in absolute value than the absolute values of the stresses already in the material, it is expected that the pulse will move in a direction to lower the strain energy per unit volume. If a small compressive pulse is in a region of material that is tensile, for example, and the pulse arrives at a region that is compressive, the pulse is expected in this analysis to reflect. An incoming tensile pulse would be expected to transmit into the region of material that is under compression.

If the incoming pulse is much larger than either of the stresses in the regions where it could reflect or transmit, it is expected that a small portion of the incoming stress, with an absolute value near those in the material, would reflect a portion $\mathrm{p}_{1}$ given by Eq. 41 or 42 , depending upon whether the incoming pulse is shear or longitudinal. The rest of the incoming pulse would continue to transmit through the material.

\section{Discussion}

The form of the pulse in Eq. 39 predicts that tight transitions between tension and compression over short distances in the material are important in affecting the transmission and reflection of the pulse. This becomes apparent in the following analysis.

Equation 39 shows that the coefficients in the tensor representing longitudinal pulses have terms that are spatial derivatives of $P$. Moreover, as shown in Sect. 3, the term $v t$ in the function $P$ can be written as $x \sigma(x) / \sigma_{\infty}$. Thus when calculating the entire derivative $P^{\prime}(x)$ in Eq. 39, for example, the derivative of the term $x \sigma(x) / \sigma_{\infty}$ by itself with respect to $x$ must be zero, if it is desired to have $P^{\prime}(x)$ not increase or oscillate without bound as $x$ gets large. As shown in Sect. 3, setting the derivative of $x \sigma(\mathrm{x}) / \sigma_{\infty}$ equal to zero gives abrupt transition in the material stress $\sigma(\mathrm{x})$ for reflection and transmission of the pulse.

It is interesting to calculate the length over which transitions between tensile and compressive stresses might occur so that an electron could possibly harness the strain energy from lattice restoring forces to avoid inelastic scattering from atomic vibrations. The energy of an electron that is coupled to the dilatation 
$\Delta$ of a lattice during inelastic scattering is given approximately by $C \Delta$, where $C$ is about $1 \times 10^{-19} \mathrm{~J}$ (Kittel 1986). The angle of rotation $\varepsilon$ of a grain from which alternating tensile and compressive stresses arise is about $10^{-5}$ radians (Muskhelishvili 1953; Kennefick 2008). Suppose that $\Delta$, the fractional volume change during inelastic scattering of an electron, is also about $10^{-5}$.

Denoting an alternating tensile and compressive stress in the $x$ direction as $\mathrm{T}_{11}{ }^{\mathrm{m}}$, suppose $\mathrm{T}_{11}{ }^{\mathrm{m}}$ is about $1 \mathrm{MPa}$. The strain energy per unit volume that might be harnessed through restoring forces would be about $(1 / 2 E)\left(\mathrm{T}_{11}{ }^{\mathrm{m}}\right)^{2}$. The Young's modulus $E$ can be assigned a value of about $400 \mathrm{GPa}$, in the range of a modulus for a ceramic material. When the strain energy is equated with $C \Delta$, the volume of material needed to have the strain energy the same order of magnitude as $C \Delta$ would be about $8 \times 10^{-15} \mathrm{~m}^{3}$, or a cube of about $10 \mathrm{~nm}$ on a side. The grains or particles themselves that could provide a length scale of about $10 \mathrm{~nm}$ in a pattern of alternating tensile and compressive stresses could be about two to ten times this length (Muskhelishvili 1953; Kennefick 2005, 2008).

\section{Conclusions}

1. For general propagation of a wave, a component of its vector potential will be a function of rotations induced the lattice planes of the material.

2. Reflection of the wave can involve rotations in the incoming wave being dependent upon both in-plane and out-of-plane rotations already in the material.

3. When the incoming wave and material displacement already present are divided into scalar and vector potentials, conditions for transmission of the wave predict a relation between the scalar potential in the material and the rotation in the material in a plane perpendicular to the scalar potential.

4. For transmission, a few relations could hold between rotations of the incoming wave and those of the material. One is that rotation in a particular plane for the material must be 180 degrees out of phase with rotation in the same plane caused by the incoming wave. Another is that rotational displacements of the incoming wave are equal in magnitude to those in the material in the same plane and in planes perpendicular to the rotational displacement of the incoming wave.

5. For short distances through a material lattice, the acceleration of a pulse due to restoring forces in the material lattice predict that abrupt transitions in the magnitude of stress in the material will maximize reflection and transmission of the pulse.

6. When the stress tensor of a pulse is derived from initially large displacements due to a disturbance, the longitudinal pulses in the stress tensor, if they are not increase or oscillate without bound, also predict optimum reflection and transmission in areas of rapid change in the magnitude of stress in the material.

7. When minimizing the local strain energy per unit volume in a material lattice is used as a boundary condition for reflection and refraction of a pulse, abrupt transitions in tension and compression are again predicted to promote reflection and transmission to the greatest extent.

Acknowledgement Support for part of this work from the Air Force Office of Scientific Research under award FA 955006-0351 is gratefully acknowledged.

\section{References}

Boyce, W.E., DiPrima, R.C.: Elementary Differential Equations, pp 42-45. John Wiley and Sons, New York (1977)

Blumenfeld, R.: Dynamics of fracture propagation in the mesoscale: theory. Theor. Appl. Fract. Mech. 30(3), 209223 (1998). doi:10.1016/S0167-8442(98)00061-5

Chattopadhyay, A.: Wave reflection and refraction in triclinic crystalline media. Arch. Appl. Mech. 73(8), 568-579 (2004). doi:10.1007/s00419-003-0308-3

Cominou, M., Dundurs, J., Chez, E.L.: Total reflection of SH waves in the presence of slip and friction. J. Acoust. Soc. Am. 66, 489-793 (1979)

Gastaldi, D., Vena, P., Contro, R.: Hybrid microstructural finite element modelling for intergranular fracture in ceramic compostes and coated systems. Comput. Mater. Sci. (2008, in press)

Geng, J., Reydellet, G., Clement, E., Behringer, R.P.: Green's functions measurements of force transmission in 2D granular materials. Physica D 182(3-4), 274-303 (2003). doi:10.1016/S0167-2789(03)00137-4

Kennefick, C.M.: Transmission of elastic stress through circular and elliptic cross sections of microstructural elements embedded in a matrix material. J. Appl. Mech. 72(4), 558-563 (2005). doi:10.1115/1.1935525 
Kennefick, C.M.: Elastic stresses in three dimensions due to the rotation of a grain or particle embedded in a material. J. Mech. Mater. Struct. (2008, in review )

Kinra, V.K., Maslow, K., Henderson, B.K., Diderich, G.: The transmission of a longitudinal wave through a layer of spherical inclusions with a random or periodic arrangement. J. Mech. Phys. Solids 46(1), 153-165 (1998). doi: 10.1016/S0022-5096(97)00030-6

Kinra, V.K., Henderson, B.K., Maslov, K.I.: Elastodynamic response of layers of spherical particles in hexaganoal and square periodic arrangements. J. Mech. Phys. Solids 47(10), 2147-2170 (1999). doi:10.1016/S0022-5096(99) 00013-7

Kittel, C.: Introduction to Solid State Physics, 6th edn, pp. 637-639. John Wiley and Sons, Inc., New York (1986)

Krasil'nikov, V.A.: Nonlinear acoustics of condensed media: history and development. Acoust. Phys. 45(3), 376-382 (1999)

Miller, R.K., Tran, H.J.: Reflection, refraction and absorption of elastic waves at a fractional interface: SH motion. J. Appl. Mech. 46, 625-630 (1979)

Miller, R.K., Tran, H.J.: Reflection, refraction and absorption of elastic waves at a fractional interface: $\mathrm{P}$ and $\mathrm{SV}$ motion. J. Appl. Mech. 48, 155-160 (1981)
Muskhelishvili, N.I.: Some Basic Problems of the Mathematical Theory of Elasticity, pp. 349-351. P. Noordhoff Ltd., Goningen (1953)

Nadeau, G.: Introduction to Elasticity, p. 35, 51. Holt, Rinehart and Winston, Inc., New York (1964)

Shim, V.P.W., Guo, Y.B., Lan, R.: Elastic stress transmission in cellular systems - analysis of wave propagation. Int. J. Impact Eng. 35, 845-869 (2008). doi:10.1016/j.ijimpeng. 2008.01.001

Singh, R.P., Kavaturu, M.: Initiation, propagation and arrest of an interface crack subjected to controlled stress wave loading. Int. J. Fract. 83(3), 291-304 (1997). doi:10.1023/ A: 1007358901588

Tumar, S.K., Khurana, A.: Elastic waves in an electro-microelastic solid. Int. J. Solids Struct. 45, 276-302 (2008)

Wang, Y.S., Dai, H.H., Yu, G.L.: Nonlinear interaction of an elastic pulse with a frictional contact interface between two anisotropic dissimilar media. J. Vib. Acoust. Trans. ASME 126(1), 108-117 (2004) 